\title{
The miR-27a-calreticulin axis affects drug-induced immunogenic cell death in human colorectal cancer cells
}

\author{
T Colangelo ${ }^{1}$, G Polcaro ${ }^{1}$, P Ziccardi ${ }^{1}$, L Muccillo ${ }^{1}$, M Galgani ${ }^{2}$, B Pucci ${ }^{3}$, M Rita Milone ${ }^{3}$, A Budillon ${ }^{3}$, M Santopaolo ${ }^{4}$, G Mazzoccoli ${ }^{5}$, \\ G Matarese $^{2,4}$, L Sabatino ${ }^{1}$ and V Colantuoni ${ }^{*, 1}$
}

Immunogenic cell death (ICD) evoked by chemotherapeutic agents implies emission of selected damage-associated molecular patterns (DAMP) such as cell surface exposure of calreticulin, secretion of ATP and HMGB1. We sought to verify whether miR-27a is implicated in ICD, having demonstrated that it directly targets calreticulin. To this goal, we exposed colorectal cancer cell lines, genetically modified to express high or low miR-27a levels, to two bona fide ICD inducers (mitoxantrone and oxaliplatin). Low miR-27a-expressing cells displayed more ecto-calreticulin on the cell surface and increased ATP and HMGB1 secretion than high miR-27a-expressing ones in time-course experiments upon drug exposure. A calreticulin target protector counteracted the miR-27a effects while specific siRNAs mimicked them, confirming the results reported. In addition, miR-27a negatively influenced the PERK-mediated route and the late PI3K-dependent secretory step of the unfolded protein response to endoplasmic reticulum stress, suggesting that miR-27a modulates the entire ICD program. Interestingly, upon chemotherapeutic exposure, low miR-27a levels associated with an earlier and stronger induction of apoptosis and with morphological and molecular features of autophagy. Remarkably, in ex vivo setting, under the same chemotherapeutic induction, the conditioned media from high miR-27aexpressing cells impeded dendritic cell maturation while increased the secretion of specific cytokines (interleukin (IL)-4, IL-6, IL-8) and negatively influenced $\mathrm{CD}^{+} \mathrm{T}$-cell interferon $\gamma$ production and proliferation, all markers of a tumor immunoevasion strategy. In conclusion, we provide the first evidence that miR-27a impairs the cell response to drug-induced ICD through the regulatory axis with calreticulin.

Cell Death and Disease (2016) 7, e2108; doi:10.1038/cddis.2016.29; published online 25 February 2016

Normal cells culminate their life span with a death process that has been proposed to occur in at least three major types. Apoptosis is a well-defined process of programmed cell death that includes both an extrinsic and intrinsic pathway and is characterized by cell shrinkage and fragmentation of cellular components including DNA that leads to the formation of apoptotic bodies efficiently cleared by phagocytes. Necrosis usually occurs in pathological circumstances and is characterized by disruption of the cell membrane, swelling of the cytoplasm, breakdown of mitochondria and DNA degradation. All cellular components are released in the extracellular environment where they act as danger signals to promote inflammation. ${ }^{1-3}$ Autophagy is primarily an evolutionarily conserved pathway involving the degradation of cellular components. In detail, autophagy is initiated with the formation of autophagosomes engulfed with cytosolic materials, fusion with lysosome to form autolysosomes followed by degradation to simple components to meet the energetic and anabolic needs of the cell. Autophagy is then a stress response system required for survival, although in cancer it has a dual role acting either as a tumor suppressor or an oncogene in a context-dependent manner. ${ }^{3,4}$ Apoptotic, necrotic and autophagic tumor cells release damage-associated molecular patterns (DAMP) which are recognized by receptors on the surface of immune cells largely determining whether cell death is immunogenic (immunogenic cell death, ICD) or tolerogenic (tolerogenic cell death). ${ }^{5,6}$ This topic is gaining increasing moment in anticancer therapy as resistance to apoptotic cell death has been recognized as a major hallmark of cancer affecting the tumor phenotype and its progression. Specifically, a selected class of chemotherapeutic agents (anthracyclines and radiations) elicit an active anti-tumor response through emission of DAMPs such as ecto-calreticulin, ATP and HMGB1 secretion in a process defined drug-induced ICD that is part of the cellular unfolded protein response (UPR) to endoplasmic reticulum (ER) stress stimuli. ${ }^{5-8}$ Once exposed, in addition to apoptosis and/or autophagy, DAMPs serve as signals to facilitate the engulfment of dying cells by macrophages and dendritic cells (DCs), leading to the activation of a potent anticancer immunity. ${ }^{9,10}$ Among the emitted DAMPs,

\footnotetext{
${ }^{1}$ Department of Sciences and Technologies, University of Sannio, Benevento 82100, Italy; ${ }^{2}$ Istituto di Endocrinologia e Oncologia Sperimentale, Consiglio Nazionale delle Ricerche (IEOS-CNR), Napoli 80131, Italy; ${ }^{3}$ Centro Ricerche Oncologiche Mercogliano, Istituto Nazionale Tumori Fondazione G. Pascale - IRCCS, Mercogliano (AV) 83013, Italy; ${ }^{4}$ Dipartimento di Medicina Molecolare e Biotecnologie Mediche, Università di Napoli "Federico II", Napoli 80131, Italy and "Division of Internal Medicine and Chronobiology Unit, IRCCS - "Casa Sollievo della Sofferenza" Hospital, San Giovanni Rotondo (FG) 71013, Italy

${ }^{*}$ Corresponding author: V Colantuoni, Department of Sciences and Technologies, University of Sannio, Via Port'Arsa 11, Benevento 82100, Italy. Tel: +390824305102; Fax: +390824305147; E-mail: colantuoni@unisannio.it

Abbreviations: CRC, Colorectal cancer; ICD, Immunogenic cell death; DAMP, damage-associated molecular patterns; UPR, unfolded protein response; ER, endoplasmic reticulum

Received 22.12.15; accepted 05.1.16; Edited by G Melino
} 
calreticulin is gaining interest because of its pleiotropic functions: it acts, in fact, as an ER chaperone, is implicated in the protein-loading complex to assemble the mature $\mathrm{MHC}$ class I molecules on the cell surface, activates the apoptotic pathway and, upon ICD inducer administration, translocates to the cell surface where it acts as an 'eat me' signal to mount an efficient immune response. Reduction of ecto-calreticulin exposure greatly impairs the apoptotic pathway and the immune response in vitro and in vivo. ${ }^{11,12}$

microRNA dysregulation is a common feature of human malignancies as they act as onco-miRNAs or tumor suppressor miRNAs; their implication in the immune response is under active investigation. ${ }^{13-15}$ We have identified miR-27a as one of the upregulated miRNAs in CRC and proved that it acts as an oncomiRNA as it is already overexpressed in adenomas and further increases during tumor progression. ${ }^{16}$ miR-27a is a member of the miR-23a, miR-27a and miR-24-2 cluster and is acquiring a growing regulatory role because it is implicated in multiple processes including tumorigenesis. ${ }^{17,18}$ We have also demonstrated that calreticulin is a direct target of miR-27a and mediates $\mathrm{MHC}$ class I molecules expression. ${ }^{16}$ We sought to verify whether miR-27a can modulate the events related to cell death and drug-induced ICD. Here, we provide evidence that miR-27a impairs the kinetics of calreticulin surface exposure along with ATP and HMGB1 secretion following mitoxantrone or oxaliplatin treatment. A selective calreticulin target protector counteracted while specific siRNAs mimicked the miR-27aspecific effects supporting the pivotal role played by this protein. miR-27a hampered the cellular UPR route following exogenous ER stress stimuli. Finally, miR-27a impaired the apoptotic and autophagy pathways, suggesting that it is a master regulator in determining cell fate. In ex vivo setting, under the same chemotherapeutic induction, the conditioned media (CM) from high miR-27a-expressing cells impeded DC maturation while increased secretion of specific cytokines (interleukin (IL)-4, IL-6, IL-8) and negatively influenced CD4 ${ }^{+}$ T-cell interferon $\mathrm{Y}$ production and proliferation, markers of a tumor immunoevasion strategy.

\section{Results}

miR-27a down-modulates emission of DAMPS upon ICD inducers. Cell death is the most enriched pathway in the Ingenuity Pathway Analysis generated from the list of differentially expressed proteins (red elements = upregulated proteins; green elements=downregulated proteins) after miR-27a silencing in HCT116 cells (Figure 1a). ${ }^{16}$ Having shown that calreticulin is a target of $\operatorname{miR}-27 a^{16}$ and given its pivotal role also in ICD, we assessed the effects of miR-27a on ecto-calreticulin, ATP and HMGB1 release (i.e., DAMPs emission) from dying cells undergoing anticancer drug-induced ICD. ${ }^{4,6,10,19}$ We exposed HCT116 CRTL, miR27a_KD and miR27a_OE cells to the anthracycline mitoxantrone (MTX, $1 \mu \mathrm{M})$ or oxaliplatin (OXP, $100 \mu \mathrm{M})$, two bona fide ICD inducers, in time-course experiments and DAMPs exposure/release was analyzed. In flow cytometry, both drugs induced a slow but significant calreticulin translocation to the cell surface in HCT116 cells, that was modest in miR27a_KD and even less evident in miR27a_OE cells, likely owing to the silenced or forced miR-27a expression, respectively, coupled with the fact that calreticulin is a miRNA direct target (Figure $1 \mathrm{~b}$ and Supplementary Figure S1A). These results were confirmed by immunofluorescence staining of non-permeabilized cells, showing the typical 'patches' of ecto-calreticulin, especially in miR27a_KD, and analysis of the isolated plasma membrane fraction (Figures $1 \mathrm{c}$ and d). ${ }^{12,20}$ Of note, also the secreted calreticulin, already present in basal conditions, increased, especially in miR27a_KD, in line with its higher plasma membrane content, indicating that miR-27a also modulates calreticulin secretion (Figure 1e). We also evaluated two additional DAMP (ATP and HMGB1) release during druginduced ICD. ${ }^{19,21}$ The kinetics of ATP release in the cell culture media showed that cells with a silenced miR-27a were more responsive to both drugs and secreted higher amounts of ATP; opposite results were obtained in cells overexpressing miR-27a (Figure 2a). Intracellular ATP mirrored the extracellular amount in all cell lines investigated (Supplementary Figure S1B). Finally, time-course of HMGB1 release evidenced in miR27a_KD that the protein, already secreted in basal conditions, slightly increased upon exposure to both drugs; in HCT116 CTRL, the increase was delayed, even further in miR27a_OE cells (Figures $2 b$ and d).

The kinetics of DAMP emission upon MTX or OXP administration was investigated in an additional CRC-derived cell line (RKO) and the derived miR27a_KD and miR27a_OE cell clones having miR-27a silenced or overexpressed, respectively. An earlier and robust calreticulin translocation to the cell membrane was detected in miR27a_KD, slower and marginal in miR27a_OE and intermediate in parental RKO cells. Also, the time-course of ATP and HMGB1 release showed the same behavior with a response that was inversely related to miR-27a levels (Supplementary Figures S2A and C). That similar results were obtained with two distinct drugs in different $\mathrm{CRC}$ cell lines indicates that miR-27a modulation of DAMP emission following chemotherapeutic stimulation is a general phenomenon and that, according to its levels, miR-27a sensitizes the cells finely tuning the response. The central role that calreticulin has in this pathway was further demonstrated in transfection assays with a specific target protector: a marked enrichment of the protein was detected on the surface of HCT116 CTRL and miR27a_OE, likely owing to higher miR-27a levels (Supplementary Figures S3A and B). In contrast, transfection of three independent siRNAs reduced ecto-calreticulin in all cells, in proportion to the different protein content, as reported. ${ }^{16}$ Interestingly, transfection of calreticulin target protector stimulated secretion of ATP and HMGB1 in HCT116 and miR27a_OE cells, while the specific siRNAs reduced secretion of both compounds in all cell lines (Supplementary Figures S3C and D).

miR-27a dictates the decision between cell death and survival. Antitumor agent-induced ICD is associated with the activation of programmed cell death pathways. ${ }^{2,6}$ To verify that miR-27a influences apoptosis, we performed timecourse experiments of MTX treatment on HCT116 and derived clones. Both the cleaved forms of PARP and caspase 3 , already detectable in basal conditions in miR27a_KD, further increased starting from earlier times with respect to 
a

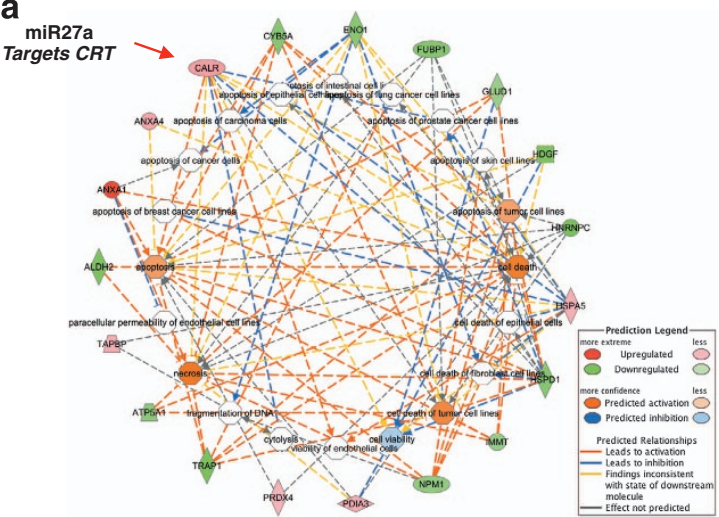

b

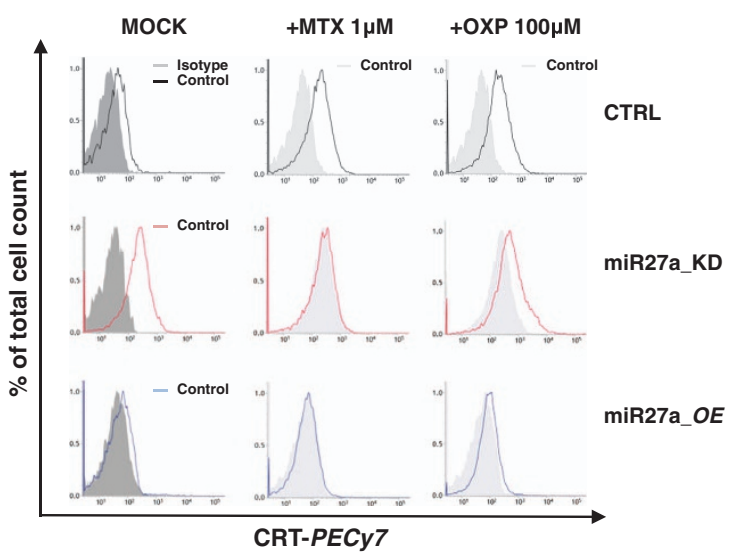

C

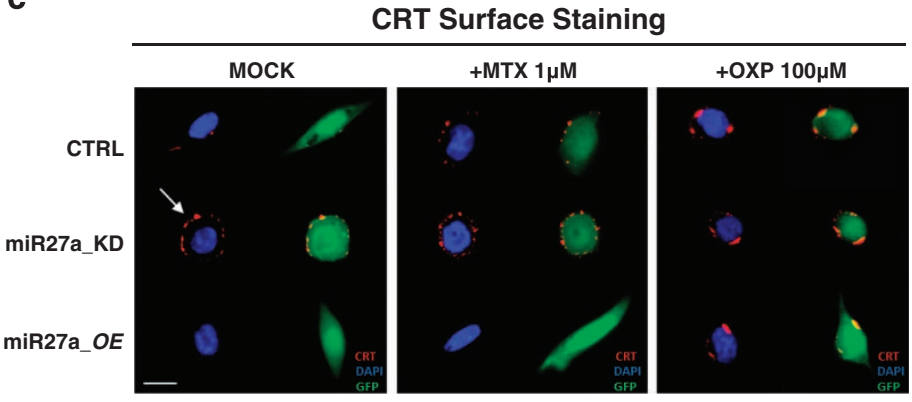

d

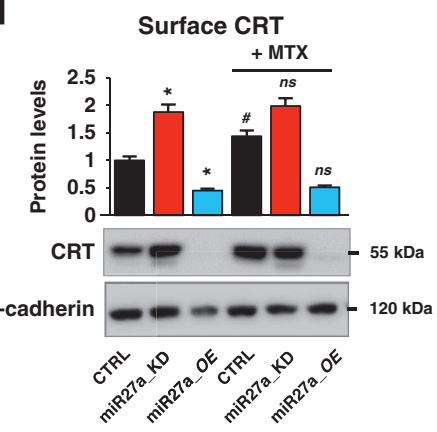

e Extracellular

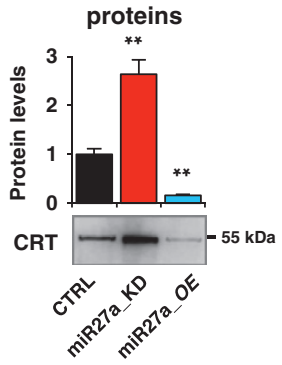

Figure 1 Calreticulin cell surface exposure is downregulated by miR-27a. (a) Cell deaths were the most enriched networks in the Ingenuity Pathway Analysis generated from the list of differentially expressed proteins (red elements = upregulated proteins; green elements = downregulated proteins) after miR-27a silencing in HCT116 cells. ${ }^{16}$ (b) Cellsurface calreticulin (CRT) assessed by flow cytometry or (c) immunofluorescence staining or (d) western blot in the isolated plasma membrane fraction from HCT116 CRTL, miR27a_KD and miR27a_OE cells exposed to mitoxantrone (MTX, $1 \mu \mathrm{M}$ ) or oxaliplatin (OXP, $100 \mu \mathrm{M})$ for $12 \mathrm{~h}$. (CRT = red; nuclei = blue; GFP = green as a marker). The white arrow indicates the patches of ecto-CRT. (Scale bar, $5 \mu \mathrm{m}$ ). Positivity for E-cadherin, a plasma membrane protein, proved that the identified proteins were truly integral membrane components in (d). Immuno-detection of extracellular CLR in the culture media of HCT116 CRTL, miR27a_KD and miR27a_OE. The histogram shows the relative quantification of the bands. Samples were analyzed in triplicate and data are mean \pm S.D. and representative of three experiments in $(\mathbf{b}, \mathbf{d}) .{ }^{*} P \leqslant 0.05$; ${ }^{*} P \leqslant 0.01$ (two-tailed Student's $t$-test)

HCT116 and miR27a_OE in which the increase was delayed (Figures $3 a$ and $b$ ). Similar results were obtained in RKO and corresponding clones (Supplementary Figure S4A). Timecourse experiments were also carried out with OXP in both cell lines and derived clones. By flow cytometry, low miR-27aexpressing cells displayed an increase of the pre-apoptotic (annexin $\mathrm{V}-\mathrm{PE}^{+}$and $7-\mathrm{AAD}^{-}$) and apoptotic (annexin $\mathrm{V}-\mathrm{PE}^{+}$ and $7-\mathrm{AAD}^{+}$) cell populations at earlier times than high miR-27a-expressing ones (Figure $3 \mathrm{c}$ and Supplementary Figure S4B).

ATP and HMGB1 release and ecto-calreticulin exposure have been associated with execution of autophagy. ${ }^{7,22,23}$ In miR27a_KD cells, this pathway is already active in basal conditions as documented by the presence of the mature LC3-II form and acquisition of autophagic morphological features (large bodies, multiple or multilobated nuclei, cytosolic vacuoles and granules) with respect to HCT116 CTRL and miR27a_OE cells (Figures $4 a$ and b). MTX administration induced autophagy in time-course experiments, as the mature LC3-II form rapidly increased in miR27a_KD and only scantly in HCT116 and miR27a_OE cells (Figure 4c). To verify that the higher LC3-II levels reflect an increased autophagic flux that culminates into the formation of autophagosomes, we tested the effects of the lysosomotropic agent, chloroquine. The mature LC3-II form showed a remarkable dose-dependent increase in miR27a_KD with respect to parental and even more than miR27a_OE cells (Figure $4 b$ ).

miR-27a hampers the chemotherapeutic-induced ICD through the same route of the UPR. Next, we asked whether miR-27a impairs drug-elicited ICD through the same route of the UPR that is activated in response to ER stimuli. ICD is, in fact, executed through activation of the doublestranded RNA-activated protein kinase-like ER kinase (PERK)-governed arm of the UPR and the PI3K-dependent secretory pathway. ${ }^{1,8,24-26}$ The kinetics of activation of this pathway was assessed: upon treatment, phosphorylation of PERK and its downstream factor elF2a, already high in miR27a_KD cells, further increased; in miR27a_OE, phosphorylation was lower and delayed with respect to HCT116 CRTL cells (Figure 5a). GRP78, another protein associated with ER stress induction, showed no significant variations 
a

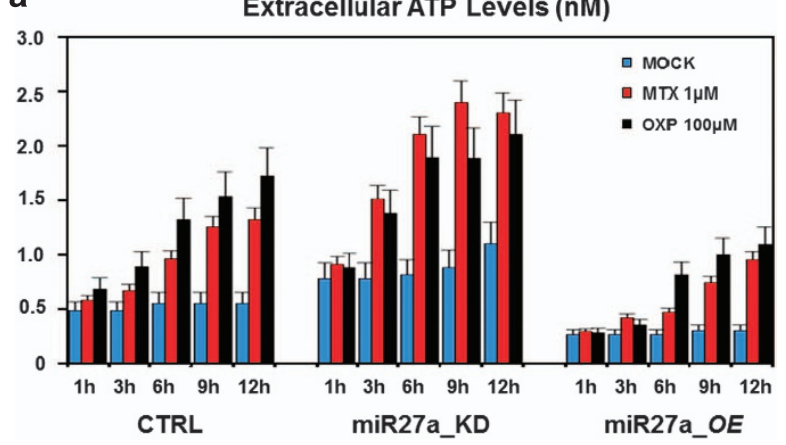

b

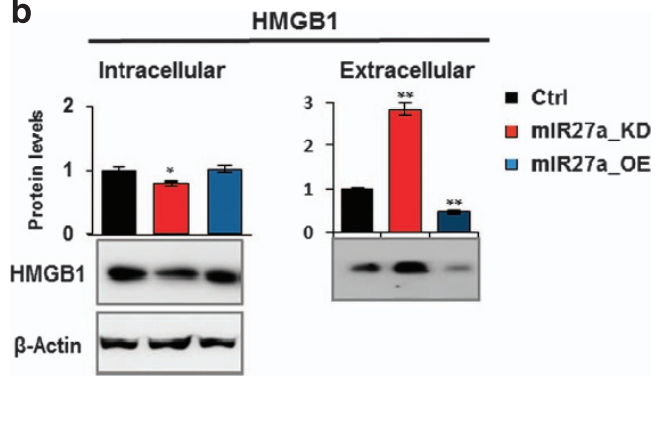

d Extracellular HMGB1 (rel.protein levels)

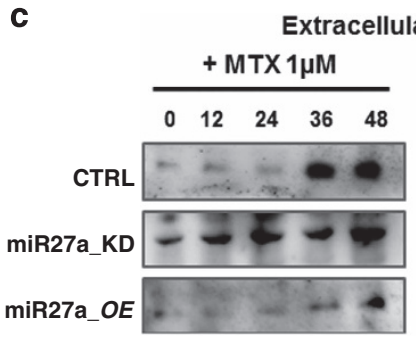
Extracellular HMGB1 \begin{tabular}{lllllll}
\multicolumn{4}{c}{ OXP } & $100 \mu \mathrm{M}$ & \\
\hline 0 & 12 & 24 & 36 & 48 & Time (h)
\end{tabular}

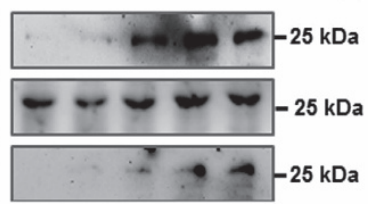
口 MTX
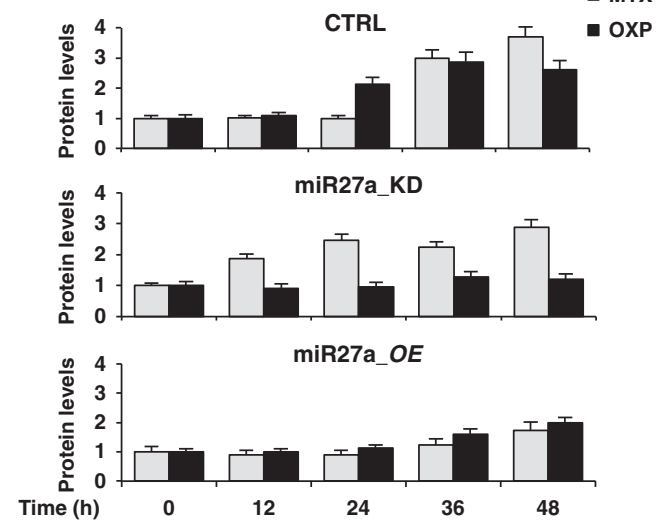

Figure 2 miR-27a affects the kinetics of emission/release of DAMPs upon chemotherapeutic-mediated ICD. (a) Kinetics of induction of ATP secretion in HCT116 CRTL, miR27a_KD and miR27a_OE cells exposed to MTX $(1 \mu \mathrm{M})$ or OXP $(100 \mu \mathrm{M})$. (b) Immuno-detection of intracellular and extracellular HMGB1 in HCT116 CRTL, miR27a_KD and miR27a_OE cells; the quantification of the bands is illustrated in the corresponding histograms. (c) Time-course of HMGB1 secretion upon exposure of HCT116 CRTL, miR27a_KD and miR27a_OE cells to either MTX or OXP quantified as reported in the histograms in (d). All values are mean \pm S.D. of three independent experiments. ${ }^{*} P \leqslant 0.05$; ${ }^{* *} P \leqslant 0.01$ (two-tailed Student's $t$-test)

with respect to the differential basal levels (Figure 5a). The other two arms of the UPR were tested and found not affected, as previously reported ${ }^{8}$ (our data not shown). The ability of miR-27a to modulate the PI3K-dependent late secretory step was proved by assessing the amount of ectocalreticulin in the plasma membrane fraction before and after administration of the PI3K inhibitor LY-294002 alone or in combination with MTX. Phosphorylation of AKT, a protein target of PI3K, was almost undetectable in HCT116 and derived clones demonstrating the efficacy of the treatment (Supplementary Figure S5A). LY-294002 caused a remarkable impairment of calreticulin plasma membrane translocation in miR27a_KD, less evident in HCT116 and miR27a_OE cells, likely owing to the higher miR-27a levels. The combined treatment did not rescue ecto-calreticulin in all cells (Figure 5b). Collectively, miR-27a affects the magnitude of DAMP emission in response to MTX through the same UPR route, according to its endogenous levels.

miR-27a in CRC cells influences DC maturation, proliferation and IFN- $\gamma$ production by $\mathrm{CD}^{+} \mathrm{T}$ cells in ex vivo experiments. We investigated whether miR-27a affects the ability of DAMPs emitted in response to chemotherapeutics to act as immunogenic signals towards monocyte-derived immature DCs prompting their maturation. ${ }^{4,6,27}$ HCT116 cells were transiently transfected with a specific miR-27a mimic (S), antisense (AS) or scrambled control RNA (C), treated with MTX or OXP for the last $12 \mathrm{~h}$ and co-cultured with human immature DCs (hu-iDCs) for further $20 \mathrm{~h}$ (Figure 6a). In flow cytometry experiments, expression of the DC maturation antigens (MHC class II/CD86; CD80/CD83) ${ }^{19}$ increased in co-cultures of hu-iDCs with AS-transfected and treated tumor cells with respect to S- or C-transfected and treated cells. Stimulation of DCs with lipopolysaccharide was used as the positive control (Figure 6b). Similar results were obtained with co-cultures of hu-iDCs with transfected and treated RKO cells (Supplementary Figure S5B) and with HCT116 and RKO stable-derived clones (data not shown). The CM from S-, ASor C-transfected cells alone or from co-cultures with hu-iDCs were assessed for a cytokine array (Figure 6c). IL-8 was detected in the $\mathrm{CM}$ from S-transfected cells alone and co-cultures with S- and C-transfected cells indicating that it was related to miR-27a expression levels. IL-4 was only detected in the co-cultures media, in particular in those from S- and C-transfected HCT116 cells. In addition, IL-6 was found only in the $\mathrm{CM}$ from co-cultures with S-transfected HCT116 cells (Figure 6d). As engulfment of mature DCs with dying tumor cells is able to mount an active immune 
a

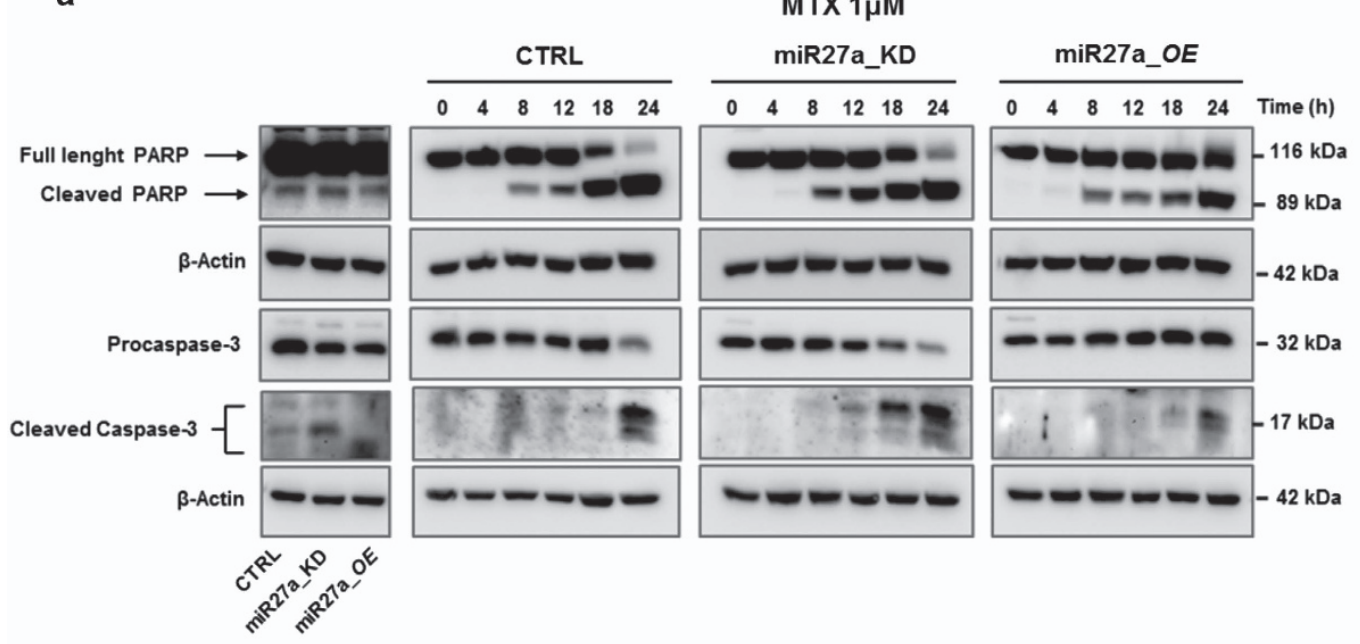

b

Cleav. PARP

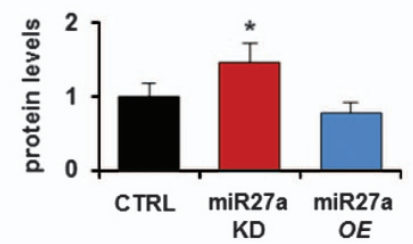

Cleav. Caspase 3

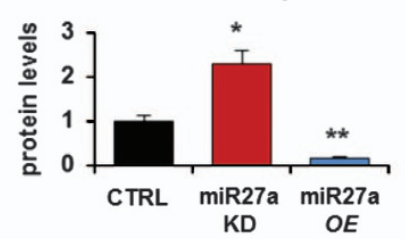

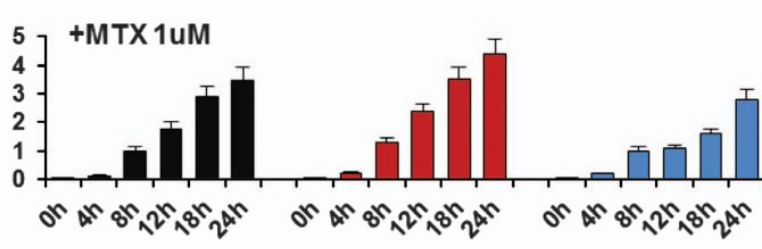

Cleav. Caspase 3

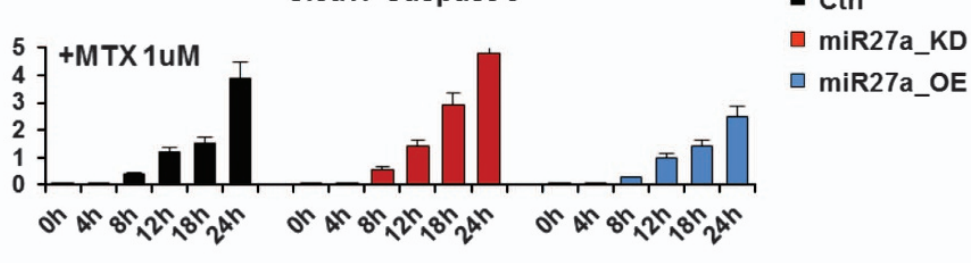

miR27a_KD

- miR27a_OE

C OXP $100 \mu \mathrm{M}$
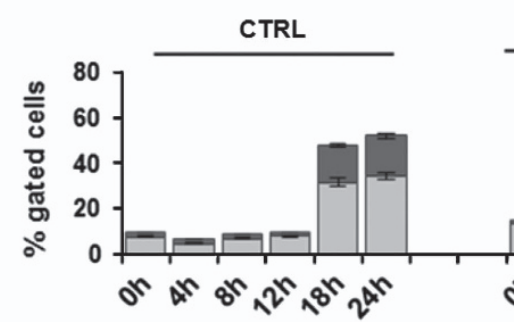

miR27a_KD
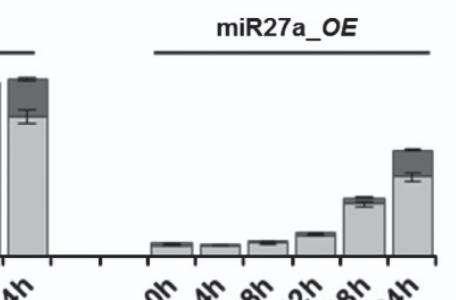

Annexin V+

7-AAD-

Annexin $\mathrm{V}+$

7-AAD+

Figure 3 miR-27a impairs the kinetics of apoptosis execution in drug-induced ICD. (a) Western blot analysis of the apoptosis markers, PARP and caspase 3, in time-course experiments of HCT116 CRTL, miR27a_KD and miR27a_OE cells treated or not with mitoxantrone (1 $\mu \mathrm{M})$. $\beta$-Actin was used as loading control and quantification of the cleaved protein forms is reported in (b). (c) Flow cytometry analysis of pre-apoptotic (annexin V-PE ${ }^{+}$and 7-AAD) and apoptotic (annexin V-PE ${ }^{+}$and 7-AAD ) HCT116 CRTL, miR27a_KD and miR27a_OE cells treated with OXP $(100 \mu \mathrm{M})$ in kinetics experiments. One representative experiment out of three is shown

response, ${ }^{6,19}$ the $\mathrm{CM}$ from AS-transfected HCT116 cells were tested and found to stimulate proliferation of naive freshly isolated $\mathrm{CD}^{+} \mathrm{T}$ cells more than those from $\mathrm{S}$ - or C-transfected cells (Figure 7a). The induction was stronger in T-cell receptor stimulated (anti-CD3/anti-CD28) CD4 ${ }^{+} \mathrm{T}$ cells displaying the same correlation with miR-27a levels. ${ }^{28}$ The CMs from co-cultures of hu-iDCs with the same cells pretreated with MTX or OXP exhibited the same trend either on unstimulated and T-cell receptor-stimulated $\mathrm{CD}^{+} \mathrm{T}$ cells
(Figure 7b). Finally, the CMs from co-cultures of hu-iDCs with AS-transfected and pretreated cells promoted higher IFN- $\gamma$ production from unstimulated $\mathrm{CD}^{+} \mathrm{T}$ cells than $\mathrm{CMs}$ from co-cultures of $\mathrm{C}$ - and remarkably S-transfected cells; as expected, the effect was stronger in T-cell receptor-stimulated $\mathrm{CD}^{+} \mathrm{T}$ cells (Figure 7c). Taken together, these results indicate that, in response to antitumor drugs, miR-27a in CRC cells impairs immunogenic signal emission and DC maturation, whereas stimulates secretion of cytokines associated 

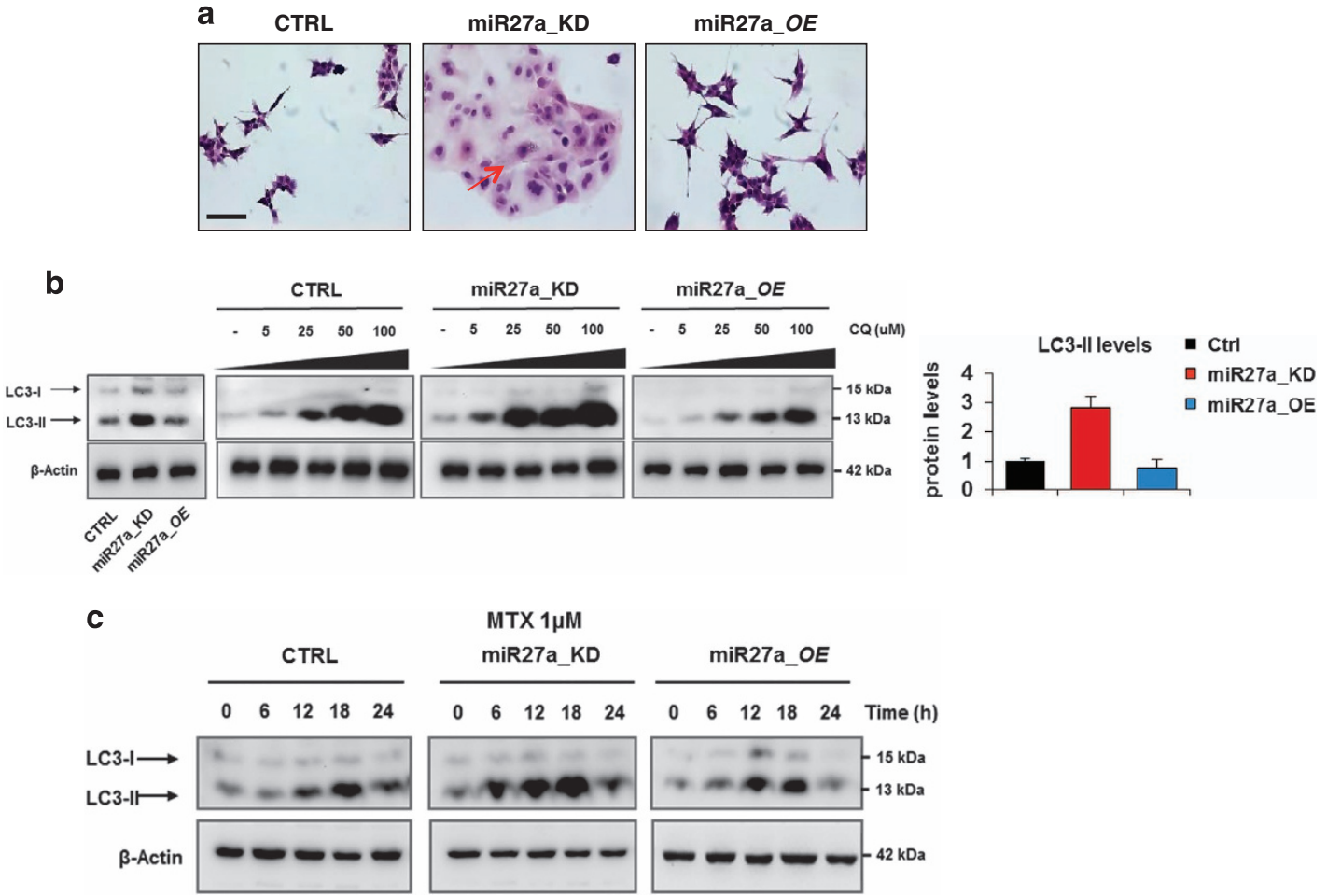

Figure 4 miR-27a knockdown promotes autophagy in CRC cells. (a) Morphological features of HCT116 CRTL, miR27a_KD and miR27a_OE cells were evaluated by hematoxylin and eosin staining; the arrows point out some remarkable phenotypic characteristics (Scale bar, $20 \mathrm{~mm}$ ). (b) Dose-dependent induction of autophagy in HCT116 CRTL, miR27a_KD and miR27a_OE cells exposed to the lysosomotropic drug chloroquine (CQ). Western blot analysis of the LC3-II mature form, an autophagic marker, is reported along with its relative quantification in the histogram. $\beta$-Actin was used as loading control. (c) Time-course of autophagy induction in HCT116 CRTL, miR27a_KD and miR27a_OE cells upon MTX treatment at $1 \mu \mathrm{M}$. Immunoblot of LC-3-II was used as a marker. All data are representative of three independent experiments and error bars represent S.D. of technical replicates (mean \pm S.D.)

with CRC progression, ${ }^{29-31}$ influences activation and IFN- $\gamma$ production from $\mathrm{CD} 4^{+} \mathrm{T}$ cells suggesting a possible role in eliciting an active immune response.

\section{Discussion}

Living organisms retain their homeostasis through a finely tuned balance between cell proliferation and death. Multiple modes of cell death exist in nature, and apoptosis or type 1 programmed cell death is likely the most frequent both in normal and tumor cells and characterized by typical morphological changes. ${ }^{1-3}$ Apoptosis has also been considered as a non-ICD mechanism, although in the past years, this concept has been revised: in fact, several anti-neoplastic drugs such as anthracyclines, oxaliplatin or hypericin-based photodynamic therapy and oncolytic coxsackievirus B3 (CVB3) trigger immunogenic apoptosis. ${ }^{32,33}$ This conceptual change stems from the observation that apoptotic cancer cells treated with these chemotherapeutics or radiotherapy release a series of DAMPs that could provoke an immune response. ${ }^{8,9,10,34}$ The hallmarks of ICD are calreticulin cell surface exposure, ATP and HMGB1 secretion. Here, we provide evidence that in CRC-derived cell lines exposed to two different bona fide ICD inducers (mitoxantrone and oxaliplatin), the amount of calreticulin translocated onto the cell surface is greatly impaired in cells overexpressing miR-27a, and heightened in miR-27a-silenced cells. We have recently identified calreticulin as a direct target of miR-27a and the effect reported here is the result of such modulation. ${ }^{16}$ The effect appears to be highly specific as a calreticulin target protector or specific siRNAs produce similar results, supporting the central role that the protein has in this pathway in line with data from the literature. ${ }^{11,12,35}$ Interestingly, high miR-27aexpressing cells exhibit reduced ATP secretion and HMGB1 release; whether these effects are due to the control of miR-27a or other still uncovered genes or non-coding RNAs is not known at the moment. We demonstrate that their secretion parallel ecto-calreticulin exposure with a similar early and strong induction in conditions of low miR-27a levels, suggesting that miR-27a silencing sensitizes the cells, tuning the response to chemotherapeutic agents. ${ }^{35,36}$

That miR-27a modulates the entire ICD program is further supported by the investigation of the UPR route. ICD is, in fact, executed through activation of the PERK-governed arm and the PI3K-dependent secretory pathway of the UPR to ER stress. ${ }^{1,24,25,26}$ We show that miR-27a greatly impairs the entire pathway especially the late secretory module; silencing the microRNA rescues the entire arm, suggesting that ATP and/or HMGB1 secretion might be regulated through the same route as previously suggested; ${ }^{4}$ elucidation of this topic, however, requires deeper and more targeted experiments.

High miR-27a-expressing cells display enhanced cell growth and survival potential, whereas its silencing stimulates the apoptotic pathway, indicating that miR-27a dictates the cell 
a

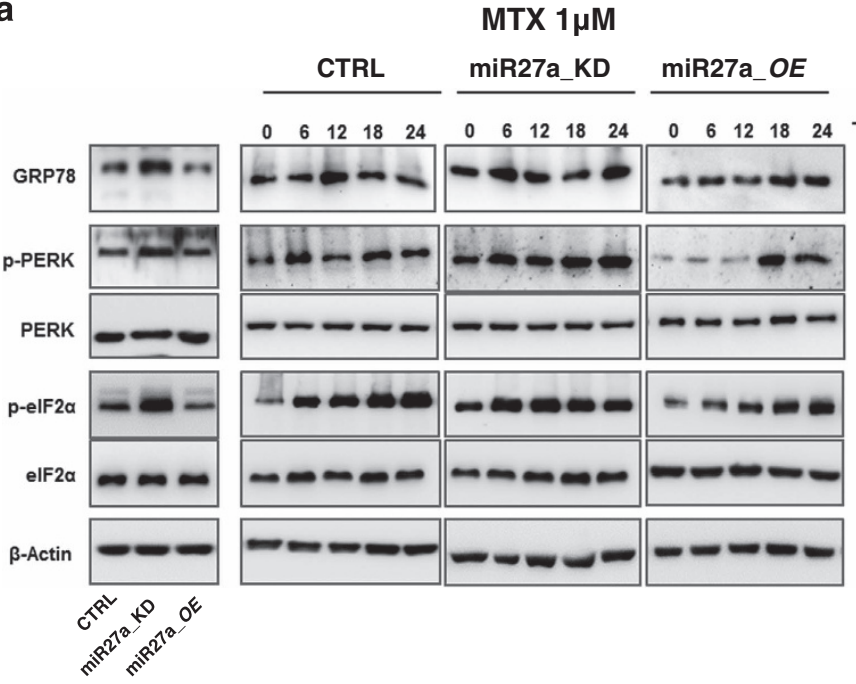

CTRL

miR27a_KD
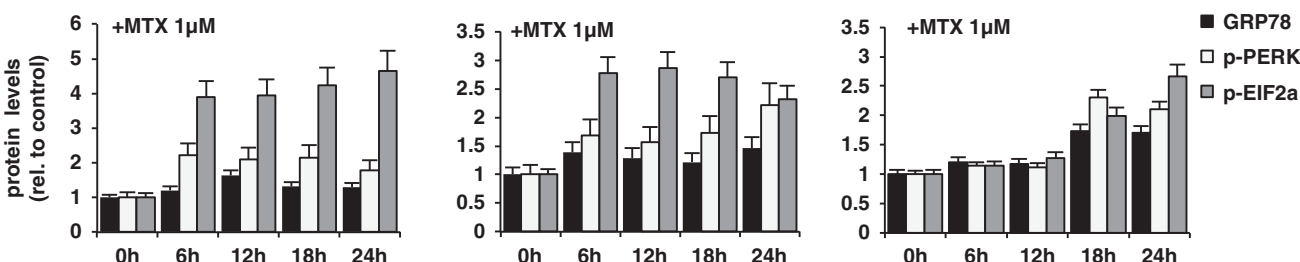

b

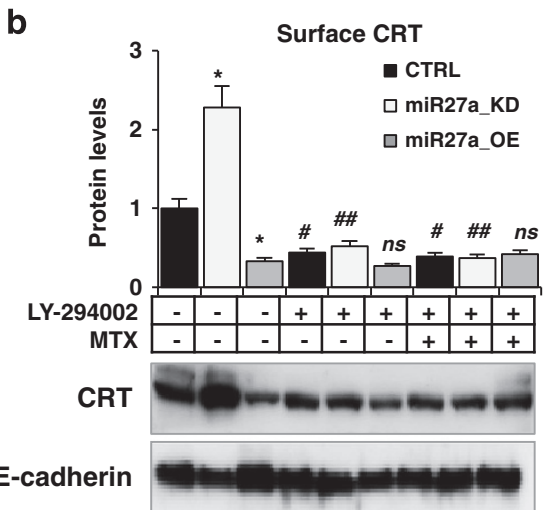

Figure 5 miR-27a counteracts mitoxantrone-induced ICD through the same UPR pathway. (a) Immunoblot analysis of PERK, p-PERK, elF2 $\alpha$, p-elF2 $\alpha$ and GRP78 in timecourse experiments of HCT116 CRTL, miR27a_KD and miR27a_OE cells treated with $1 \mu \mathrm{M} \mathrm{MTX;} \mathrm{phosphorylation} \mathrm{was} \mathrm{referred} \mathrm{to} \mathrm{equivalent} \mathrm{amounts} \mathrm{of} \mathrm{the} \mathrm{non-phosphorylated}$ forms of the proteins in all cells and then to $\beta$-actin used as a loading control. Quantification of the bands in basal and in the kinetic conditions is reported. (b) CRT cell surface

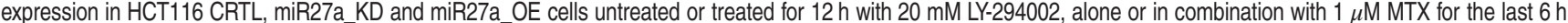
Densitometric analysis was carried out after normalization to E-cadherin. ${ }^{*} P \leqslant 0.05 ;{ }^{* *} P \leqslant 0.01$ (two-tailed Student's $t$-test) versus HCT116 CRTL cells; ${ }^{\#} P \leqslant 0.05 ;{ }^{* \# \#} P \leqslant 0.01$ (two-tailed Student's t-test) versus MTX-untreated cells. The values are representative of five experiments

decision between survival and death in vitro and in mouse xenografts supporting the conclusion that miR-27a acts as an oncomiRNA. ${ }^{16}$ Remarkably, we find that miR-27a inversely modulates also autophagy and that, in our cell model, apoptosis and autophagy operate in the same direction. ${ }^{3,4}$ As ATP and HMGB1 have been associated with execution of autophagy, collectively, our data are consistent with an overall ICD process repression influenced by miR-27a.

DAMP emission has been associated with the ability to elicit an efficient immune response. In fact, ecto-calreticulin acts as an 'eat me' signal, secreted ATP as a 'find me 'signal and released HMGB1 as a docking site for TLR and other receptors on the surface of dendritic and phagocytic cells. ${ }^{5,7}$ We demonstrate here in ex vivo experiments that CRC cells expressing low levels of miR-27a subject to drug-induced ICD elicit efficient maturation of DC cells and cytokine secretion, induce proliferation of $\mathrm{CD}^{+} \mathrm{T}$ cells and INF- $\gamma$ secretion, all markers of immune activation. Full validation of an efficient anti-tumor response requires experiments in immunocompetent mice models; nonetheless, the analysis of our series of sporadic CRCs shows that high miR-27a-expressing tumors associate with reduced ecto-calreticulin, $\mathrm{CD}^{+}$and $\mathrm{CD}^{+}$ 

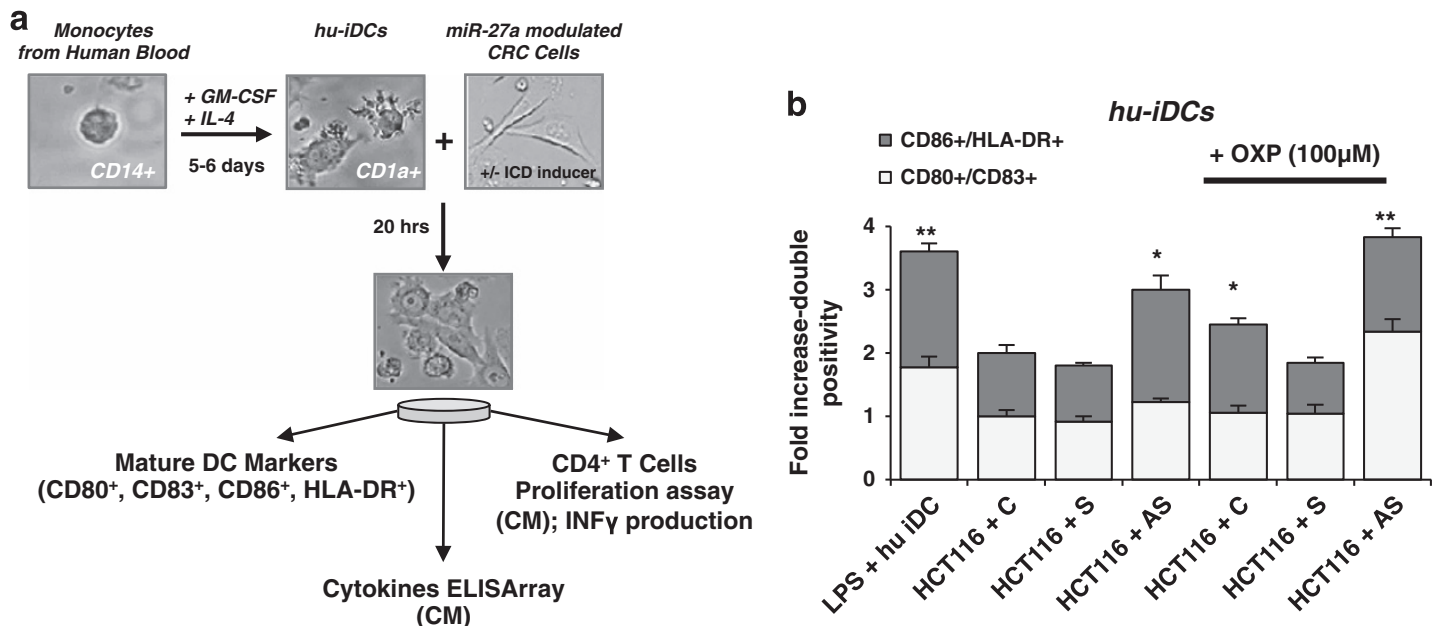

C

Cytokines ELISArray

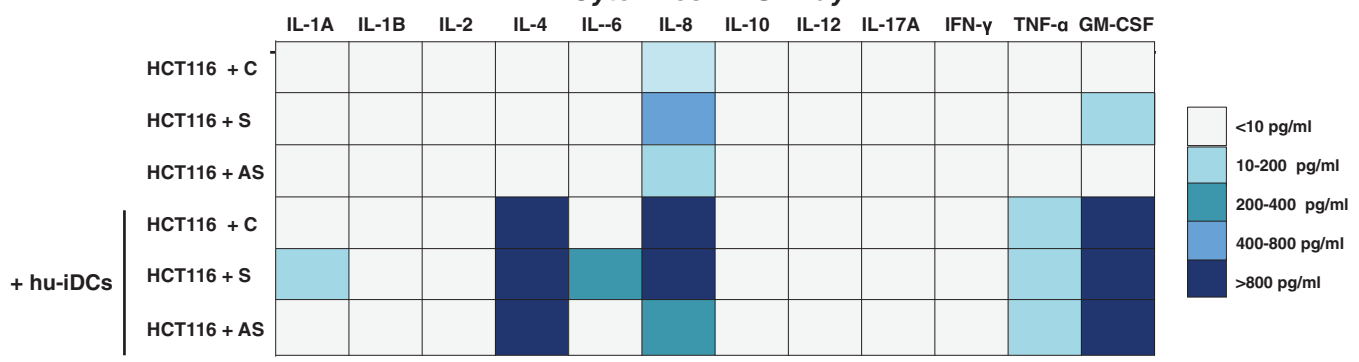

d

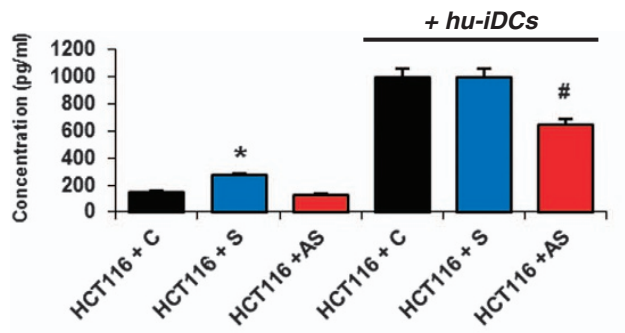

IL-4

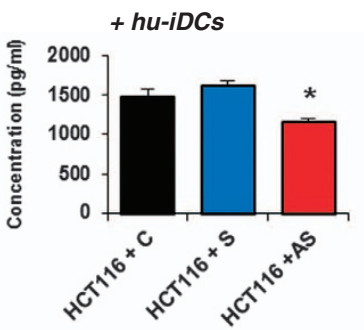

IL-6

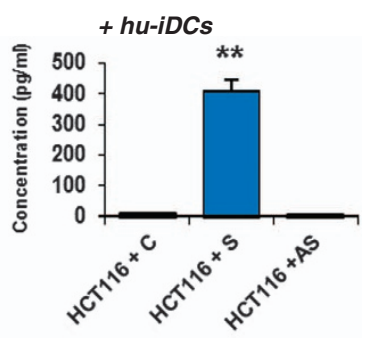

Figure 6 miR-27a in CRC cells modulates monocyte-derived human DCs maturation and secretion of selected cytokines upon drug-mediated ICD. (a) Scheme of the experimental setting: hu-iDCs were generated from filtered buffy coat-derived monocytes donated by healthy donors and grown for $5 / 6$ days in the presence of specific stimulatory factors. Hu-iDCs were identified by FACS analysis of CD1a ${ }^{+}$cells, counted and co-cultured (1:2 ratio) with HCT116 or RKO cells previously transfected for $72 \mathrm{~h}$ with a miR-27a synthetic mimic (S), AS or scrambled control (C) RNA, respectively, and treated for the last $12 \mathrm{~h}$ with OXP $(100 \mu \mathrm{M})$. (b) Flow cytometry analysis of specific DC maturation markers $\left(\mathrm{CD} 6^{+} / \mathrm{HLA}-\mathrm{DR}{ }^{+}, \mathrm{CD} 80^{+} / \mathrm{CD} 83^{+}\right)$upon $20 \mathrm{~h}$ of co-cultures as described in (a); lipopolysaccharide stimulation $(0.1 \mathrm{mg} / \mathrm{ml})$ of hu-iDCs was used as positive control of DC maturation. Only the results with OXP treatment are reported, similar results were obtained with MTX. The histogram illustrates the results of five experiments. (c) A cytokine ELISA array was performed on the CM from S-, AS- or C-transfected HCT116, alone or from co-cultures of the same cells with hu-iDCs; the color-code reports the range of values of the cytokines detected. (d) The histogram shows quantification of IL-8, IL-4 and IL-6 detected with the cytokine ELISA array. Samples were analyzed in triplicate and data are mean \pm S.D. and representative of three experiments. ${ }^{*} P \leqslant 0.05 ;{ }^{* *} P \leqslant 0.01$ (two-tailed Student's $t$-test)

infiltration and activity. ${ }^{16}$ Interestingly, the combination high $\mathrm{miR}-27 \mathrm{a} / \mathrm{low}$ calreticulin is linked to liver metastasis and a worse prognosis, further implying the role that the miR-27acalreticulin axis may have in stimulating the immune system and thus in the overall survival of CRC patients. ${ }^{16}$ Of note, calreticulin seems to be the dominant determinant in this analysis. ${ }^{35,36}$ Our data are supported by a recent study in which a reduced level of calreticulin in a naturally occurring cancer cell model is the primary, if not the only reason, to explain resistance to anticancer vaccination. Moreover, calreticulin levels are predictive of patient clinical responses to ICD-inducing anticancer therapies with possibly clinical implications. In this ICD clinical setting, calreticulin positively correlates with the expression of phagocytosis-associated genes relevant for phagosome maturation/processing. ${ }^{9}$

The existence of mechanisms of resistance to anticancer vaccination effect represents a major obstacle for antitumor immunotherapy, so that their identification could explain the failure of certain subsets of patients in responding to ICD inducers. ${ }^{9}$ The results we present here unveil miR-27a as a negative regulator of the drug-induced ICD response by downregulating calreticulin expression and cell surface 

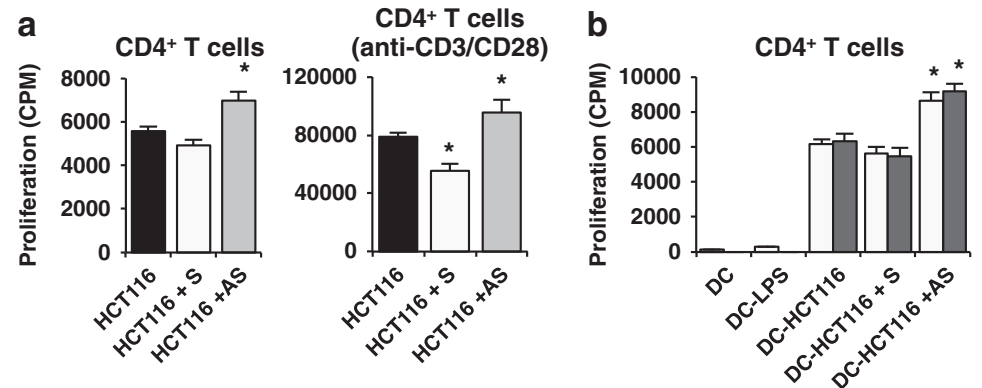

C

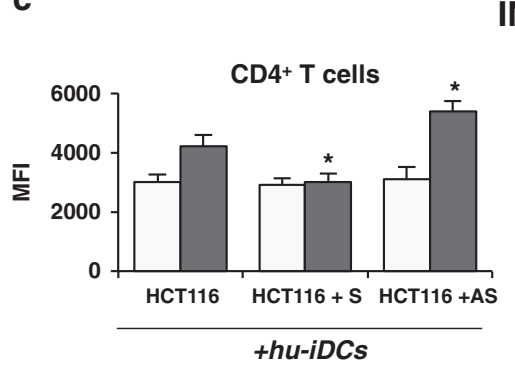

INFY
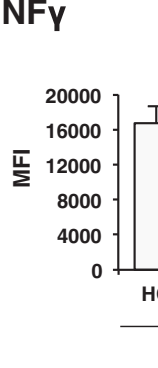
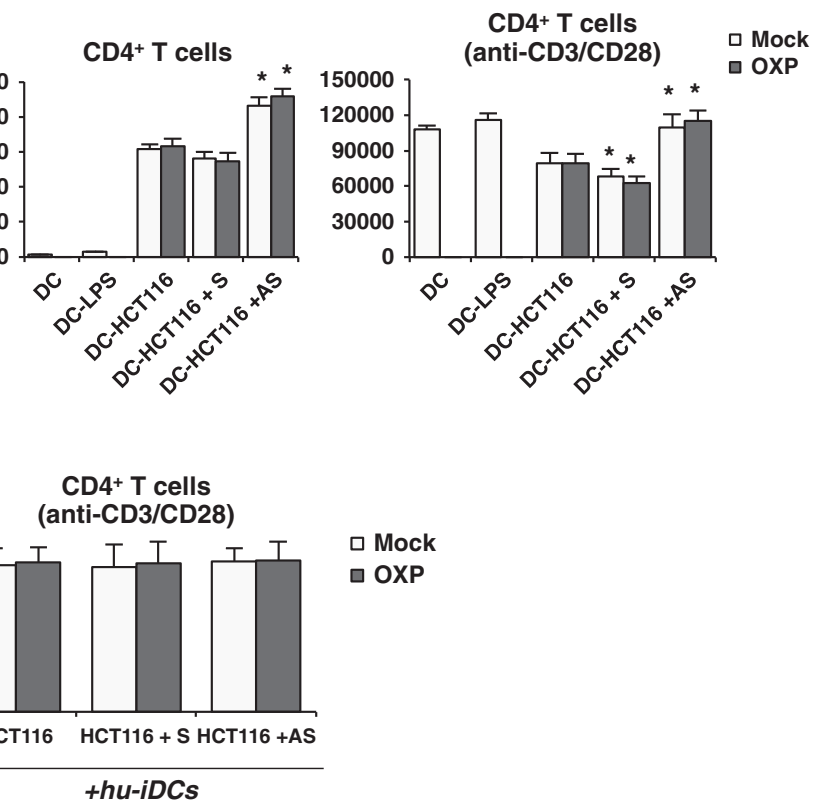

Figure 7 miR-27a influences proliferation and IFN- $\gamma$ production by CD4 ${ }^{+}$T cells in ex vivo experiments upon drug-mediated ICD. The CM from S-, AS- or C-transfected and treated HCT116 alone (a) or from co-cultures of the same transfected and pretreated cells cells with hu-iDCs (b) were added to naïve, freshly isolated CD4 ${ }^{+} \mathrm{T}$ cells either unstimulated or stimulated with anti-CD3/CD28 dynabeads to test their ability to proliferate. Samples were analyzed in triplicate and data are mean \pm S.D. and representative of three experiments. ${ }^{*} P \leqslant 0.05$ (two-tailed Student's $t$-test). (c) To analyze the production of IFN- $\gamma$, freshly isolated $\mathrm{CD}^{+} \mathrm{T}$ cells were cultured overnight in the presence or absence of anti-CD3/CD28 dynabeads and exposed to CMs from co-cultures as in (b). Samples were analyzed in triplicate and data are mean \pm S.D. and representative of three experiments. ${ }^{*} P \leqslant 0.05 ;{ }^{*} P \leqslant 0.01$ (two-tailed Student's $t$-test)

exposure. Calreticulin, thus, has a critical position at the interface between cancer cells and the immune system. ${ }^{12,35,36}$ The disclosure of the miR-27a-calreticulin axis and its role in ICD may pave the way for future research aimed to stratify CRC patients on the basis of miR-27a/calreticulin expression levels and design novel personalized therapeutic interventions.

\section{Materials and Methods}

Cell culture. Human colon cancer cell lines HCT116 and RKO were purchased from American Type Culture Collection (ATCC, Rockville, MD, USA) and cultured as reported. $^{37}$

Oligonucleotides and plasmid transfection. Synthetic miR-27a mimic (Syn-hsa-miR-27a), miR-27a inhibitor (anti-hsa-miR-27a) or the appropriate scrambled controls (AllStar or mirScript Inhibitor-Negative Control) were purchased from Qiagen (Hilden, Germany). The miR-27a-antisense (MZIP27a-PA-1), the premiR-27a expression constructs (PMIRH27a-onlyPA-1) and scrambled control miRNAs (MZIP000-PA-1; PMIRH000PA-1) plasmids (System Biosciences, Mountain View, CA, USA) were transfected in the different CRC cell lines. microRNA functional studies were performed by inhibiting miRNA-mRNA target interactions either with a custom-designed calreticulin-miScript Target Protector or a negative control miScript Target Protector (MTP0075035; Qiagen). Detection of no variations in unrelated proteins validated target protector specificity. A gene-specific package of three preselected siRNAs against calreticulin (Flexi Tube siRNA GS811) or a negative control siRNA (SI03650325) (Qiagen) was used in transient transfections. Functional assays, RNA and protein analyses were performed within $24 / 72 \mathrm{~h}$ from transfections. In each experiment, the extent of miR-27a silencing/overexpression was assessed by qRT-PCR. Plasmids and oligonucleotides were transfected using Lipofectamine 2000 (Thermo Fisher, Waltham, MA, USA) and HiPerFect Transfection Reagent (Qiagen) or RNAi Max (Thermo Fisher), respectively, according to the manufacturers' recommendations.
mRNA/miRNA extraction and qRT-PCR analysis. Total RNA was extracted from cells and tissues using TRIzol (Thermo Fisher) and treated with DNase I. microRNAs were extracted using the Qiagen miRNeasy Mini Kit (Qiagen) according to the manufacturer's protocol. Assessment of RNA purity and quantity was performed as described. ${ }^{37}$

Western blot analysis. Protein extracts from cell lines and tissues were prepared and analyzed as previously reported. ${ }^{37}$ Antibodies against calreticulin (ab2907), HMGB1 (ab79823) were from Abcam (Cambridge, MA, USA); GRP78 (sc-13968), PERK (sc-377400), p-PERK (sc-32577), Caspase-3 (sc-7148), antimouse (sc-2031), anti rabbit (sc-2004) from Santa Cruz Biotechnology (Dallas, TX, USA), LC3 (GTX 82986) from Genetex (Irvine, CA, USA); $\beta$-actin (F-3022) from Sigma-Aldrich (Milan, Italy); E-cadherin (BD 610405) from BD transduction (BD Biosciences, San Jose, CA, USA); AKT (Y058027) and p-AKT (Y080084) from abm (Richmond, BC, Canada); elF2a (\#9722), p-elF2a(\#3597) and PARP (\#9542 S) from Cell Signaling (Danvers, MA, USA). To analyze surface proteins, we used an extraction method based on a published procedure. ${ }^{38}$ Positivity for E-cadherin, a plasma membrane protein, and negativity for $\beta$-actin, a cytosolic protein, proved that the identified proteins were truly integral membrane components. Coomassie blue staining was also used for assessing equivalent protein load. In some experiments, the culture medium $(2-8 \mathrm{ml})$ was collected and concentrated to feasible volumes (200-500 $\mu$ l) via centrifugation $(2000 \times g$ for $1 \mathrm{~h}$ ) through Centricon microconcentrators PLUS $10 \mathrm{KDa}$ filter (Merck-Millipore, Darmstadt, Germany). Proteins were then analyzed by western blot.

Immunofluorescence. Immunofluorescence staining was carried out on nonpermeabilized HCT116 or RKO and derivative clones exposed or not to pharmacological treatments. Cells were plated on coverslips, fixed in paraformaldehyde (4\% in PBS) at room temperature for $10 \mathrm{~min}$, blocked in bovine serum albumin ( $3 \%$ in PBS) for 30 min before incubation with specific antibodies to calreticulin (1:100 dilution) for $1 \mathrm{~h}$ at room temperature. Subsequently anti-rabbitTritc (GTXRB-003-ERHOX, 1 : 1000 dilution) (Immunoreagent, Raleigh, NC, USA) were incubated for $1 \mathrm{~h}$ at room temperature. Coverslips were washed with PBS, stained with DAPI and, after three more washes in cold PBS, mounted in mowiol 
4-88 (Merck-Millipore) on glass slides. For negative controls, only secondary antibodies were used in the absence of primary antibodies. The fluorescence signals were observed and captured using a Carl Zeiss LSM700 laser-scanning microscope (Carl Zeiss, Jena, Germany). Hematoxylin and eosin staining of CRC cells was performed and evaluated as previously reported. ${ }^{37}$ Image acquisition and analysis were performed on DM100 Leica Photosystem 40.106.206 (Leica, Milan, Italy).

Flow cytometry. Flow cytometry was used to detect calreticulin on the cell surface of HCT116 and RKO and their derivative clones mir27a_KD or mir27a_OE in basal condition, upon MTX or OXP treatment. Briefly, cells were plated, harvested and washed twice with PBS and incubated for $1 \mathrm{~h}$ in darkness at $4{ }^{\circ} \mathrm{C}$ with a custom PE-Cy-7-labeled anti-CRT, using PE/Cy7 conjugation kit (ab102903; Abcam), as manufacturer's protocol. Cells were then washed and resuspended in cold PBS for FACS analysis. Apoptosis was evaluated by AnnexinV-PE, 7-AAD using the Apoptosis Detection Kit I (BD Biosciences), according to the manufacturer's protocols. All flow cytometry results were analyzed with FACSuite Software v.1.0.5.3841 (BD Biosciences).

Pharmacological treatments. To elicit ICD, cells were treated with $1 \mu \mathrm{M}$ mitoxantrone (MTX, Sigma-Aldrich) or $100 \mu \mathrm{M}$ oxaliplatin (OXP, Sigma-Aldrich) two well-known ICD inducers, in time-course experiments. To block the secretory module, cells were incubated with $20 \mathrm{mM}$ LY-294002 (Sigma-Aldrich), a selective PI3K inhibitor, for $12 \mathrm{~h}$ alone or in combination with MTX for the last $6 \mathrm{~h}$ of the treatment. The efficacy of the treatment was proved by assessing the phosphorylation level of AKT, a downstream target of PI3K. Chloroquine diphosphate salt (CQ, Sigma-Aldrich) was added to the cells at the indicated concentrations and the amount of the mature LC3-II form assessed.

ATP assay. Extracellular and intracellular ATP levels were measured by the luciferin-based ENLITEN ATP Assay (Promega, Madison, WI, USA). The ATP-driven chemoluminescence was recorded on Luminometer TD-20/20 (Promega).

Ex vivo isolation and maturation of human DCs (hu-DC). DCs were isolated from peripheral blood mononuclear cells, obtained from $30 \mathrm{ml}$ of leukocyteenriched buffy coat from healthy donors, centrifuged with $\mathrm{F}$ Lymphoprep gradient (Axis-Shield PoC AS, Dundee, Scotland); the light density fraction was recovered. Monocytes were purified by FACS-sorting (BD Biosciences) using a phycoerythrin (PE)-anti-CD14 ${ }^{+}$antibody (BD Biosciences). Cells were then seeded at a concentration of $0.5-1 \times 10^{6}$ cells/ml in RPMl 1640 supplemented with $10 \%$ fetal calf serum or $2 \%$ human serum (Sigma-Aldrich), $25 \mathrm{mM}$ HEPES and $2 \mathrm{mM}$ glutamine (complete medium) containing $100 \mathrm{ng} / \mathrm{ml}$ granulocyte macrophagecolony-stimulating factor (Sigma-Aldrich) and $100 \mathrm{ng} / \mathrm{ml}$ IL-4 (Sigma-Aldrich) and cultured for 5-6 days to obtain a population of immature DCs (hu-iDCs), monitoring their morphology and expression of the specific surface marker CD1a. Immature DCs were scraped, counted and co-cultured for $24 \mathrm{~h}$ in a $1: 2$ ratio with HCT116 or RKO cells previously transfected for $72 \mathrm{~h}$ with a miR-27a synthetic mimic (S), AS or scrambled control (C) RNA and treated with MTX $(1 \mu \mathrm{M})$ or OXP $(100 \mu \mathrm{M})$ for the last $12 \mathrm{~h}$. The percentage of mature DCs was assessed by FACS analysis; an aliquot of these cells was exposed to lipopolysaccharide (Sigma-Aldrich) $(0.1 \mathrm{mg} /$ $\mathrm{ml}$ ), a known pathogen-associated molecular pattern-inducing stimulus, as a positive control for DC maturation. For surface marker analysis by flow cytometry, DCs were stained with the following antibodies: PE-cyanine (Cy)-7 anti-CD80, PE anti-CD86, PE-Cy-7 anti-CD83, PE anti-CD1a (all from Becton Dickinson), Allophycocyanin (APC) anti-HLA-DR (Serotec, Raleigh, NC, USA) and analyzed with the BD FACSCanto using Diva software (BD Biosciences). The cells were also stained with the corresponding PE-, PE-Cy-7 or APC-conjugated isotype-matched control antibody from BD Biosciences.

Human cytokine ELISA array. A human cytokine Multi-AnalyteELISArray Kit (Qiagen) was used to estimate cytokine secretion by HCT116 S, AS- or C-transfected cells alone or by co-cultures of the same cells with hu-iDCs. The cytokines list comprises: IL-1 $\alpha$, IL-1 $\beta$, IL-2, IL-4, IL-6, IL-8, IL-10, IL-12, IL-17A, IFN$\gamma$, TNF- $\alpha$ and granulocyte-macrophage colony-stimulating factor. The array was performed according to the manufacturer's instructions. Cytokine levels were measured by evaluating absorbance at $450 \mathrm{~nm}$ on a Microplate reader (Tecan Group Ltd., Mannedorf, Switzerland). Sampling was performed in triplicates.

$\mathrm{CD}^{+}$T-cell isolation, proliferation and IFN- $\boldsymbol{\gamma}$ production. After Ficoll hypaque (GE-Healthcare, Pittsburgh, PA, USA) gradient centrifugation, CD4 ${ }^{+}$
T cells were isolated from PBMC by negative selection using human $\mathrm{CD}^{+} \mathrm{CD} 25^{+} \mathrm{T}$ cell kit (Thermo Fisher) (cell purity $>98 \%$ by FACS analysis) and cultured $\left(1 \times 10^{6}\right.$ cells $/ \mathrm{ml}$ ) in 96-well plates (BD Biosciences) with RPMI 1640 medium supplemented with $100 \mathrm{Ul} / \mathrm{ml}$ penicillin, $100 \mu \mathrm{g} / \mathrm{ml}$ streptomycin (Thermo Fisher) and supplemented with $5 \%$ FCS (Thermo Fisher). Cells were stimulated for 3 days with an antiCD3/CD28 Dynabeads ( 0.1 bead/cell) (Thermo Fisher) in the presence or absence of the CM obtained either from co-cultures, as reported above, or from HCT116 or RKO cells transfected with the miR-27a S, AS or C RNA and treated with MTX or OXP. On the last day, cells were exposed to $\left[{ }^{3} \mathrm{H}\right]$ thymidine $(0.5 \mu \mathrm{Ci} /$ well) (GE Healthcare) and harvested $12 \mathrm{~h}$ later. Radioactivity was measured with a $\beta$-cell-plate scintillation counter (Wallac, Waltham, MA, USA). ${ }^{39}$ To analyze the production of IFN- $\gamma$, freshly isolated $\mathrm{CD} 4^{+} \mathrm{T}$ cells were cultured overnight in the presence or absence of anti-CD3/CD28 beads ( 0.1 beads/cell). To avoid cytokine extracellular export, the cultures were incubated in the presence of $5 \mu \mathrm{g} / \mathrm{ml}$ of Brefeldin-A (Sigma-Aldrich), as previously described. ${ }^{40}$ To assess the intracellular production of IFN- $\gamma$ (Becton Dickinson), cells were treated with a fixing/ permeabilization kit (Caltag, Burlingame, UK), following the manufacturer's instructions. $\mathrm{CD}^{+} \mathrm{T}$ cells were stained with APC mouse anti-human IFN- $\gamma$ and analyzed with the BD FACSCanto using Diva software (Becton Dickinson).

Statistics. All statistical analyses were performed using Statistical Package from Social Science (SPSS; version 16.0) for Windows (SPSS Inc, Chicago, IL, USA) and R/Bioconductor (Seattle, WA, USA). Data are reported as means \pm S.D., and mean values were compared using the Student's $t$ test. Results were considered statistically significant when $P \leqslant 0.05$ was obtained.

\section{Conflict of Interest}

The authors declare no conflict of interest.

Acknowledgements. This work was partially supported by a grant from AlL (Associazione italiana per lo studio dei linfomi e plasmacitomi) to VC, by grants from the Fondazione Italiana Sclerosi Multipla (FISM) n. 2012/R/11, EU Ideas Programme, ERC-StG 'menTORingTregs' n. 310496, FIRB MERIT Grant n. RBNE08HWLZ_015, and a CNR Medicina Personalizzata Grant to GM and by a grant from Juvenile Diabetes Research Foundation (JDRF) n. 1-PNF-2015-115-5-B to MG. MS is supported by the PhD Program in Medicina Molecolare e Biotecnologie Mediche, Università degli Studi di Napoli 'Federico II'. We are grateful to Prof Marco E Bianchi for generous gift of reagents, critical reading of the manuscript and discussion. We would like to thank Mariarosaria Montagna (DBBBM) for technical assistance.

\section{Author contributions}

TC, GP and PZ performed the majority of the experiments; LM and LS performed additional experiments and obtained more data; $B P, M R M$, and $A B$ contributed with the proteomic data and $\mathrm{AB}$ with valuable discussion; MG, MS and GM contributed with the ex vivo experiments, interpretation and critically intellectual content; GM provided clinical samples and discussion; VC and LS designed the bulk of the experiments, supervised the entire study and wrote the manuscript along with TC.

1. Garg AD, Martin S, Golab J, Agostinis P. Danger signalling during cancer cell death: origins, plasticity and regulation. Cell Death Differ 2014; 21: 26-38.

2. Inoue $\mathrm{H}$, Tani K. Multimodal immunogenic cancer cell death as a consequence of anticancer cytotoxic treatments. Cell Death Differ 2014; 21: 39-49.

3. Mariño G, Niso-Santano M, Baehrecke EH, Kroemer G. Self-consumption: the interplay of autophagy and apoptosis. Nat Rev Mol Cell Biol 2014; 15: 81-94.

4. Hou W, Zhang Q, Yan Z, Chen R, Zeh lii HJ, Kang R et al. Strange attractors: DAMPs and autophagy link tumor cell death and immunity. Cell Death Dis 2013; 4: e966.

5. Tesniere A, Panaretakis T, Kepp O, Apetoh L, Ghiringhelli F, Zitvogel L et al. Molecular characteristics of immunogenic cancer cell death. Cell Death Differ 2008; 15: 3-12.

6. Krysko DV, Garg AD, Kaczmarek A, Krysko O, Agostinis P, Vandenabeele P. Immunogenic cell death and DAMPs in cancer therapy. Nat Rev Cancer 2012; 12: 860-875.

7. Bianchi ME. Killing cancer cells, twice with one shot. Cell Death Differ 2014; 21: 1-2.

8. Panaretakis T, Kepp O, Brockmeier U, Tesniere A, Bjorklund AC, Chapman DC et al. Mechanisms of pre-apoptotic calreticulin exposure in immunogenic cell death. EMBO J 2009; 28: 578-590.

9. Garg AD, Elsen S, Krysko DV, Vandenabeele P, de Witte P, Agostinis P. Resistance to anticancer vaccination effect is controlled by a cancer cell-autonomous phenotype that disrupts immunogenic phagocytic removal. Oncotarget 2015; 6: 26841-26860. 
10. Kroemer G, Galluzzi L, Kepp O, Zitvogel L. Immunogenic cell death in cancer therapy. Ann Rev Immunol 2013; 31: 51-72.

11. Raghavan M, Wijeyesakere SJ, Peters LR, Del Cid N. Calreticulin in the immune system: ins and outs. Trends Immunol 2013; 34: 13-21.

12. Obeid M, Tesniere A, Ghiringhelli F, Fimia GM, Apetoh L, Perfettini $\mathrm{JL}$ et al. Calreticulin exposure dictates the immunogenicity of cancer cell death. Nat Med 2007; 13: 54-61.

13. Di Leva G, Garofalo M, Croce CM. MicroRNAs in cancer. Annu Rev Pathol 2014; 9: 287-314.

14. Hayes J, Peruzzi PP, Lawler S. MicroRNAs in cancer: biomarkers, functions and therapy. Trends in Mol Med 2014; 20: 460-469.

15. Cekaite L, Eide PW, Lind GE, Skotheim RI, Lothe RA. MicroRNAs as growth regulators, their function and biomarker status in colorectal cancer. Oncotarget 2015 (e-pub ahead of print 25 November 2015)

16. Colangelo T, Polcaro T, Ziccardi P, Pucci B, Muccillo L, Galgani M et al. Proteomic screening identifies calreticulin as a miR-27a direct target repressing MHC class I cell surface exposure in colorectal cancer. Cell Death Dis 2016 (in press).

17. Zhang Z, Liu S, Shi R, Zhao G. miR-27 promotes human gastric cancer cell metastasis by inducing epithelial-to-mesenchymal transition. Cancer Genet 2011; 204: 486-491.

18. Jahid S, Sun J, Edwards RA, Dizon D, Panarelli NC, Milsom JW et al. miR-23a promotes the transition from indolent to invasive colorectal cancer. Cancer Discov 2012; 2: 540-553.

19. Garg AD, Krysko DV, Verfaillie T, Kaczmarek A, Ferreira GB, Marysael T et al. A nove pathway combining calreticulin exposure and ATP secretion in immunogenic cancer cell death. EMBO J 2012; 31: 1062-1079.

20. Panaretakis T, Joza N, Modjtahedi N, Tesniere A, Vitale I, Durchschlag M et al. The cotranslocation of ERp57 and calreticulin determines the immunogenicity of cell death. Cell Death Differ 2008; 15: 1499-1509.

21. Garg AD, Galluzzi L, Apetoh L, Baert T, Birge RB, Bravo-San Pedro JM. Molecular and translational classifications of DAMPs in immunogenic cell death. Front Immunol 2015; 6. 588

22. Michaud M, Martins I, Sukkurwala AQ, Adjemian S, Ma Y, Pellegatti $P$ et al. Autophagydependent anticancer immune responses induced by chemotherapeutic agents in mice. Science 2011; 334: 1573-1577.

23. Ohsumi Y. Historical landmarks of autophagy research. Cell Res 2014; 24: 9-23.

24. Verfaillie T, Rubio N, Garg AD, Bultynck G, Rizzuto R, Decuypere JP. PERK is required at the ER-mitochondrial contact sites to convey apoptosis after ROS-based ER stress. Cell Death Differ 2012; 19: 1880-1891.

25. Kepp O, Menger L, Vacchelli E, Locher C, Adjemian S, Yamazaki T et al. Crosstalk between ER stress and immunogenic cell death. Cytokine Growth Factor Rev 2013; 24: 311-318.
26. Martins I, Wang Y, Michaud M, Ma Y, Sukkurwala AQ, Shen S et al. Molecular mechanisms of ATP secretion during immunogenic cell death. Cell Death Differ 2014; 21: 79-91.

27. Wang W, Groenendyk J, Michalak M. Calreticulin signaling in health and disease. Int J Biochem Cell Biol 2012; 44: 842-846.

28. Ali TH, Pisanti S, Ciaglia E, Mortarini R, Anichini A, Garofalo C et al. Enrichment of CD56 (dim) KIR+CD57+ highly cytotoxic NK cells in tumour-infiltrated lymph nodes of melanoma patients. Nat Comm 2014; 5: 5639.

29. Francipane MG, Alea MP, Lombardo Y, Todaro M, Medema JP, Stassi G. Crucial role of interleukin-4 in the survival of colon cancer stem cells. Cancer Res 2008; 68: 4022-4025.

30. Lee YS, Choi I, Ning Y, Kim NY, Khatchadourian V, Yang D et al. Interleukin-8 and its receptor CXCR2 in the tumour microenvironment promote colon cancer growth, progression and metastasis. Br J Cancer 2012; 106: 1833-1841.

31. Guo Y, Xu F, Lu T, Duan Z, Zhang Z. Interleukin-6 signaling pathway in targeted therapy for cancer. Cancer Treat Rev 2012; 38: 904-910.

32. Mellman I, Coukos G, Dranoff G. Cancer immunotherapy comes of age. Nature 2011; 480 : 480-489.

33. Dudek AM, Carg AD, Krysko DV, De Ruysscher D, Agostinis P. Inducers of immunogenic cancer cell death. Cytokine Growth Factor Rev 2013; 24: 319-333.

34. Krysko O, Løve AT, Bachert C, Vandenabeele P, Krysko DV. Many faces of DAMPs in cancer therapy. Cell Death Dis 2013; 4: e631.

35. Obeid M, Tesniere A, Panaretakis T, Tufi R, Joza N, van Endert P et al. Ecto-calreticulin in immunogenic chemotherapy. Immunol Rev 2007; 220: 22-34.

36. Peng RQ, Chen YB, Ding Y, Zhang R, Zhang X, Yu XJ et al. Expression of calreticulin is associated with infiltration of T-cells in stage III-B colon cancer. World J Gastroenterol 2010; 16: 2428-2434.

37. Colangelo T, Fucci A, Votino C, Sabatino L, Pancione M, Laudanna C et al. MicroRNA-130b promotes tumor development and is associated with poor prognosis in colorectal cancer. Neoplasia 2013; 15: 1086-1099.

38. Yazid S, Solito E, Christian H, McArthur S, Goulding N, Flower R. Cromoglycate drugs suppress eicosanoid generation in U937 cells by promoting the release of Anx-A1. Biochem Pharmacol 2009; 77: 1814-1826.

39. Masci AM, Galgani M, Cassano S, De Simone S, Gallo A, De Rosa V et al. HIV-1 gp120 induces anergy in naive T lymphocytes through $\mathrm{CD}$ 4-independent protein kinase-A-mediated signaling. J Leukoc Biol 2003; 74: 1117-1124.

40. Galgani M, De Rosa V, De Simone S, Leonardi A, D’Oro U, Napolitani G et al. Cyclic AMP modulates the functional plasticity of immature dendritic cells by inhibiting Src-like kinases through protein kinase A-mediated signaling. J Biol Chem 2004; 31: 32507-32512.

Supplementary Information accompanies this paper on Cell Death and Disease website (http://www.nature.com/cddis) 